\title{
Horizontal Ionospheric Electron Density Gradients Observed by FORMOSAT-3/COSMIC TIP: Spatial Distributions and Effects on VLF Wave Propagation at Mid-Latitudes
}

\author{
Damien H. Chua ${ }^{1,}{ }^{,}$, Kenneth F. Dymond ${ }^{1}$, Scott A. Budzien ${ }^{1}$, Clayton Coker ${ }^{1}$, and Jann-Yenq Liu ${ }^{2}$ \\ ${ }^{1}$ Space Science Division, Naval Research Laboratory, Washington, District of Columbia, USA \\ ${ }^{2}$ Institute of Space Science, National Central University, Chung-Li, Taiwan, ROC
}

Received 9 June 2007, accepted 16 January 2008

\begin{abstract}
We investigate the spatial variability of electron densities in the nightside ionosphere and its effects on very-low frequency (VLF) wave propagation using a suite of instruments from the FORMOSAT-3/Constellation Observing System for Meteorology Ionosphere and Climate (COSMIC) spacecraft. We use observations from the Tiny Ionospheric Photometer (TIP) instruments to infer the horizontal electron density gradients along each satellite track. We demonstrate that the OI $1356 \AA$ radiance measured by the TIP instruments tracks the horizontal electron density structure well with high spatial resolution and unprecedented sensitivity. Accurate measurements of the horizontal electron density gradients are important for improving retrieved electron density profiles from GPS occultation and other tomographic remote sensing techniques. The processes underlying the variability in the large-scale, nightside electron density gradients are the main drivers of ionospheric weather. TIP observations reveal significant variability in both the small and large scale structure of the nightside ionosphere. The relative intensities, relative widths, and latitudinal separation of the northern and southern ionization crests of the Appleton anomalies show a high degree of longitudinal variation. We demonstrate how the TIP observations can be used to measure the horizontal gradient of the refractive index of whistler-mode VLF waves propagating in a cold, collisionless plasma. These measurements are critical for understanding how gradients in electron density associated with ionospheric structure such as depletions and the Appleton anomalies affect VLF wave propagation through the equatorial and mid-latitude ionosphere.
\end{abstract}

Key words: Equatorial ionosphere, VLF wave propagation, Remote sensing

Citation: Chua, D. H., K. F. Dymond, S. A. Budzien, C. Coker, and J. Y. Liu, 2009: Horizontal ionospheric electron density gradients observed by FORMOSAT-3/COSMIC TIP: Spatial distributions and effects on VLF wave propagation at mid-latitudes. Terr. Atmos. Ocean. Sci., 20, 251-259, doi: 10.3319/TAO.2008.01.16.01(F3C)

\section{INTRODUCTION}

The main challenge for ionospheric specification is making the transition from a global, climatological description of the ionosphere to one that captures variations over small spatial scales and short time scales while still maintaining world-wide coverage. Small to meso-scale ionospheric structures covering length scales from tens to hundreds of kilometers are associated with perturbations in radio frequency $(\mathrm{RF})$ waves that propagate through the ionosphere. These disturbances have been observed over a broad range of frequencies. Electron density gradients associated with the equatorial Appleton anomaly strongly refract very-

\footnotetext{
* Corresponding author

E-mail:damien.chua@nrl.navy.mil
}

low-frequency (VLF) waves (James 1972; Fiszleiber et al. 1975; Cairó and Cerisier 1976). At higher frequencies, scintillation of very-high-frequency (VHF) and ultra-highfrequency (UHF) waves are known to occur in the presence of equatorial F-region irregularities (Aarons et al. 1972; Basu 1974; Basu and Basu 1985; Basu et al. 2001). Global positioning system (GPS) L-band transmissions in the 1.0 1.6 GHz frequency range also experience scintillations in the presence of ionospheric irregularities and gradients in electron density (Bhattacharyya et al. 2000; Ledvina et al. 2004; Valladares et al. 2004). Mitigating the degraded communication and navigation capabilities that these effects produce drives the need for higher fidelity characterization of ionospheric structure over a broad range of spatial scales. 
Studying the processes responsible for variability in the structure of the ionosphere is also important for understanding how the ionosphere is coupled with regions both below (the mesosphere) and above (the magnetosphere).

In this paper, we discuss how observations of far-ultraviolet (FUV) radiative recombination emissions by the FORMOSAT-3/Constellation Observing System for Meteorology Ionosphere and Climate (COSMIC) spacecraft are used to obtain precise measurements of electron density gradients in the nightside ionosphere. We also derive a relationship between the horizontal electron density gradients measured by FORMOSAT-3/COSMIC and that of the refractive index of whistler-mode waves in a cold plasma. This relationship is important for understanding how VLF wave propagation is affected by variations in the structure of the nightside ionosphere.

FORMOSAT-3/COSMIC is a six satellite constellation in low-Earth orbit carrying a suite of instruments to monitor the ionosphere (Rocken et al. 2000). The FORMOSAT-3/ COSMIC spacecraft operate at an altitude of $800 \mathrm{~km}$ in six orbit planes spaced $24^{\circ}$ apart with $72^{\circ}$ inclination and 100 minute orbital periods. The FORMOSAT-3/COSMIC spacecraft provide measurements of the nightside ionosphere with excellent spatial coverage and high time resolution. Each FORMOSAT-3/COSMIC spacecraft carries a suite of instruments including a GPS Occultation Receiver (GOX), a Tri-Band Beacon (TBB), and a Tiny Ionospheric Photometer (TIP) (Kalmanson et al. 2004). Dymond et al. (2009) demonstrate how the GOX and TIP instruments are used together for tomographic reconstruction of the electron densities in the low-latitude, nightside ionosphere. Coker et al. (2009) give a detailed characterization of both the Appleton anomalies and low-latitude, ionospheric irregularities using TIP observations. This work focuses on the measurement of horizontal electron density gradients using measurements from the TIP instruments.

Each TIP is a FUV photometer that operates in the 1310 - $1600 \AA$ passband. The primary measurement of the TIP sensors is the radiance of the OI $1356 \AA$ emission that arises in the nightside ionosphere from radiative recombination of $\mathrm{O}^{+}$and electrons:

$\mathrm{O}^{+}+\mathrm{e}^{-} \rightarrow \mathrm{O}+h v_{1356 \AA}$

The TIP instruments each have a filter wheel that allows for a number of viewing modes. Most TIP observations of the OI $1356 \AA$ emissions in the nightside ionosphere are taken with the full, open aperture (Night mode). A pinhole mask on the filter wheel allows for a factor of four reduction in the TIP sensitivities in order to observe auroral emissions and regions near the terminator that are much brighter than the nightside $1356 \AA$ airglow. A barium fluoride $\left(\mathrm{BaF}_{2}\right)$ filter allows for the measurement of FUV emissions longward of $1356 \AA$ such as the $\mathrm{N}_{2}$ LBH bands. The TIP observations of- fer high time and spatial resolution. TIP measurements are taken with a 1.14 second cadence and dark counts are obtained every 37 seconds. The TIP instruments have a $4^{\circ}$, circular field of view which yields a spatial resolution of $35 \mathrm{~km}$ at $300 \mathrm{~km}$ altitude from a FORMOSAT-3/COSMIC satellite at $800 \mathrm{~km}$ altitude. Among the six TIP sensors, the average sensitivity is approximately 470 counts $\mathrm{s}^{-1}$ Rayleigh $^{-1}$. In comparison, previously flown FUV instruments have had sensitivities ranging from about 0.1 to a few tens of counts $\mathrm{s}^{-1}$ Rayleigh ${ }^{-1}$.

\section{ELECTRON DENSITIES FROM TIP MEASUREMENTS}

The primary goal of the TIP instruments is to provide high resolution characterizations of the electron density structure in the nightside ionosphere. This includes measuring the horizontal electron density gradients at mid- and lowlatitudes. Below we describe the method by which electron density gradients are inferred from TIP measurements and demonstrate the validity of the technique.

Each TIP instrument measures a signal that can be expressed by:

$4 \pi I=\int_{0}^{Z_{s a t}} \alpha n_{e}(z) n_{O^{+}}(z) d z$

Here $4 \pi I$ is the radiance measured by each TIP instrument in units of Rayleighs, $\alpha$ is the radiative recombination rate coefficient, and $n_{e}$ and $n_{O^{+}}$are the electron and $\mathrm{O}^{+}$densities at altitude, $z$. In Eq. (2) we have ignored multiple scattering contributions to the $1356 \AA$ radiance by atomic oxygen in the thermosphere. A more detailed treatment of the OI $1356 \AA$ radiance calculation is given by Dymond et al. $(1997,2000)$. If we assume that in the F-region of the ionosphere the electron and $\mathrm{O}^{+}$densities are approximately equal, we can simplify the integral expression in Eq. (2) to yield:

$4 \pi \mathrm{I}=K(\mathrm{nmF} 2)^{2} \mathrm{H}$

$K$ encompasses all of the constants from Eq. (2), $\mathrm{nmF} 2$ is the electron density at the F-region peak, and $\mathrm{H}$ is the $\mathrm{O}^{+}$ scale height at the altitude of the $\mathrm{O}^{+}$peak. By computing the logarithmic derivative with respect to $x$, the horizontal direction along the satellite track, we can relate the horizontal gradients of the $1356 \AA$ radiance measured by TIP to the horizontal gradients in electron density:

$\frac{\partial(4 \pi I)}{4 \pi I \partial x}=\frac{2 \partial n_{e}}{n_{e} \partial x}+\frac{\partial H}{H \partial x}$

Equation (4) states that the measured horizontal gradient in 
$1356 \AA$ radiance is equal to the sum of the gradients in the electron density and the $\mathrm{O}^{+}$scale height.

\section{TESTING GRADIENT EQUATION WITH IRI MODEL}

The validity of the assumptions used to derive the simplified relation between the measured $1356 \AA$ radiance and the electron density [Eq. (3)] and the relationship between their gradients [Eq. (4)] is evaluated using output from the International Reference Ionosphere 2001 model (IRI-2001) (Bilitza 2001). The IRI-2001 model was run to simulate equinoctial conditions in the nightside ionosphere. Electron and $\mathrm{O}^{+}$densities are computed as a function of geographic latitude between $-40^{\circ}$ and $40^{\circ}$ and altitudes between $100-$ $1000 \mathrm{~km}$ at $240^{\circ} \mathrm{E}$ longitude (Fig. 1). Large horizontal gradients in the electron density are present in this model pro-

(a)

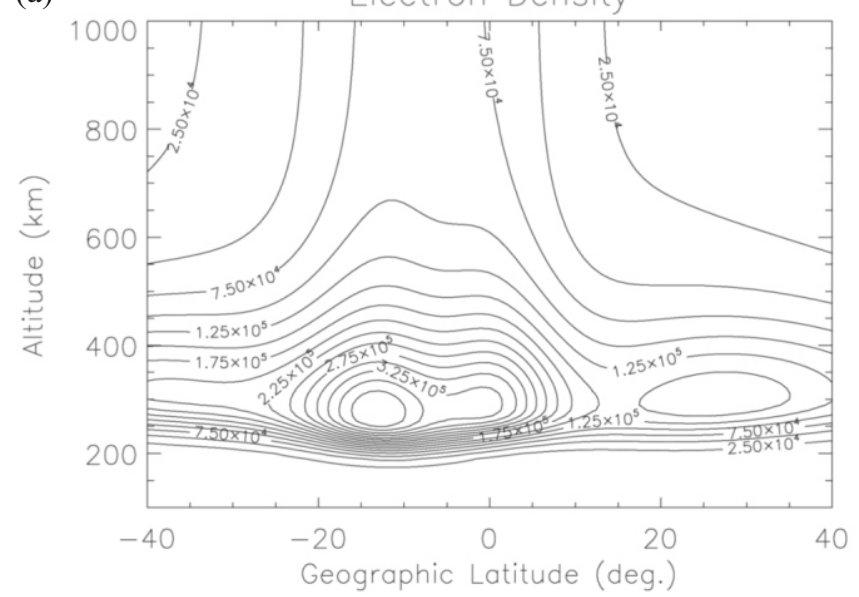

file, especially in the vicinity of the equatorial anomaly-like features near $-15^{\circ}$ and $25^{\circ}$ latitude. From the density profiles shown in Fig. 1 we compute nmF2 (Fig. 2a) and the $\mathrm{O}^{+}$scale height (Fig. 2b) as functions of geographic latitude.

The expected nadir intensity of the OI $1356 \AA$ emission from radiative recombination is computed from the density profiles shown in Fig. 1 using Eq. (2). For this calculation we use a radiative recombination rate coefficient of $7.3 \times$ $10^{-13} \mathrm{~cm}^{3} \mathrm{~s}^{-1}$ at $1160 \mathrm{~K}$ (Melendez-Alvira et al. 1999). To simulate the signal that a TIP sensor would measure in this model ionosphere, we first convert the OI $1356 \AA$ radiance to instrumental counts by assuming a sensitivity of 470 counts $\mathrm{s}^{-1}$ Rayleigh $^{-1}$. This is close to the average sensitivity among the six TIP instruments. To these synthetic TIP counts we add Poisson-distributed random counts to simulate dark noise in the instrument. The result is converted back to Rayleighs using the aforementioned sensitivity. The simu-

(b)

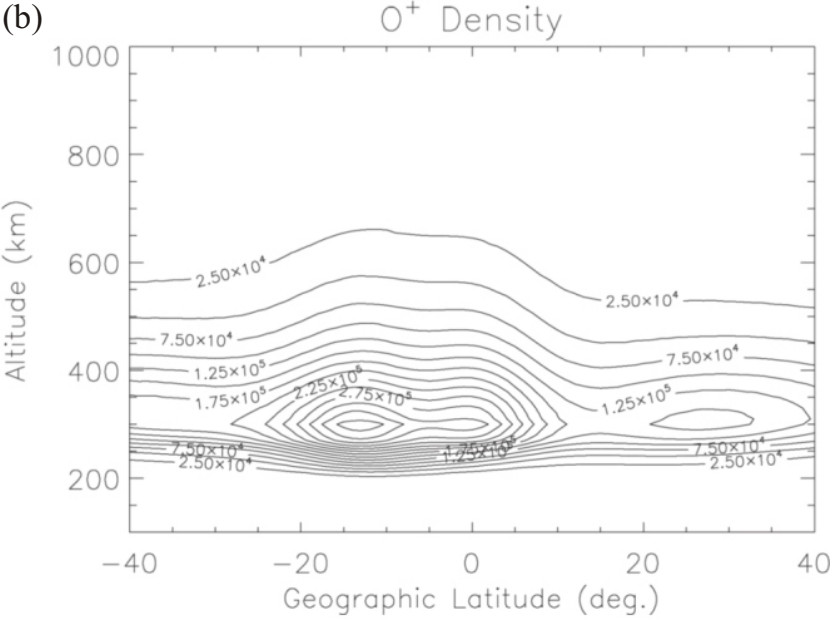

Fig. 1. (a) Electron and (b) $\mathrm{O}^{+}$density profiles output from the IRI-2001 model for equinoctial conditions in the nightside ionosphere.
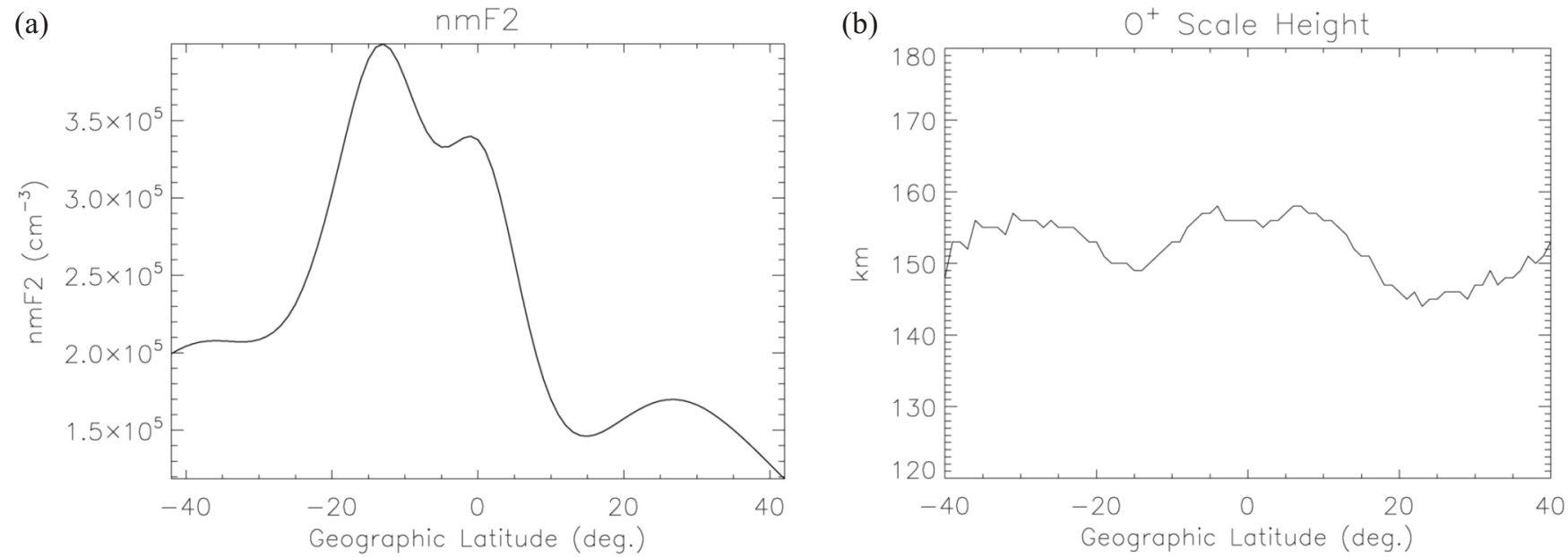

Fig. 2. $\mathrm{NmF} 2$ and the $\mathrm{O}^{+}$scale heights computed from the density profiles shown in Fig. 1. 
lated TIP OI $1356 \AA$ radiance is shown in Fig. 3.

Using the latitudinal profiles of $\mathrm{nmF} 2$, the $\mathrm{O}^{+}$scale height (Fig. 2), and the simulated OI $1356 \AA$ emission intensities (Fig. 3), we compute each term in the gradient equation [Eq. (4)] independently. Each gradient is shown separately in Fig. 4. The $n m F 2$ gradient is shown by the solid black line. The gradients in the modeled OI $1356 \AA$ intensity and the $\mathrm{O}^{+}$ scale height are shown by the dashed and dotted lines, respectively. The gradients in the OI $1356 \AA$ intensity track those in the peak electron density very well. The larger variance in the OI $1356 \AA$ intensity gradients occurs where the radiance is weak (about 0.5 Rayleighs or less) and the noise is a larger component of the simulated TIP measurement. Figure 4 also shows that the latitudinal variations in the $\mathrm{O}^{+}$scale height are small. The contribution of the $\mathrm{O}^{+}$scale height gradient to the OI $1356 \AA$ radiance variations appears to be negligible. The results from the IRI-2001 model indicate that the horizontal

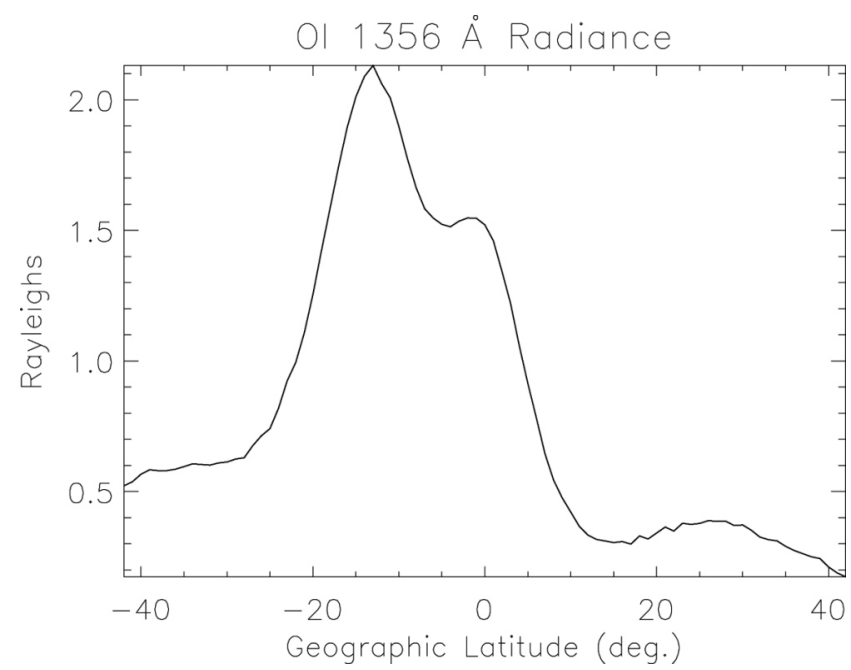

Fig. 3. Simulated OI $1356 \AA$ radiance computed from density profiles shown in Fig. 1.

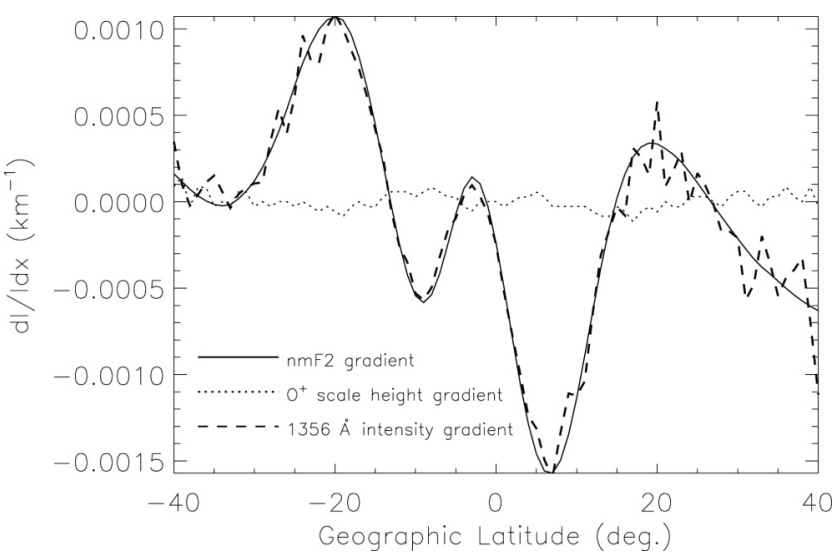

Fig. 4. Horizontal gradients of OI $1356 \AA \AA$ radiance, $\mathrm{nmF} 2$, and $\mathrm{O}^{+}$scale height. gradients in peak electron density can be characterized well by measurements of the OI $1356 \AA$ radiance alone.

\section{VALIDATING GRADIENT EQUATION WITH TIP AND GOX DATA}

To validate this technique for measuring the horizontal electron density gradients in electron density using the OI $1356 \AA$ emission, we examine observations from one of the FORMOSAT-3/COSMIC satellites (FM6) on 15 September 2006. This was a geomagnetically quiet day, with $K_{p}=0^{+}$ and $A_{p}=0$. Between $0840-0900$ UT the FORMOSAT-3/ COSMIC FM6 spacecraft is in the ascending node of its orbit in the Pacific sector about $10^{\circ}$ east of Kwajalein atoll. Figure 5 shows the OI $1356 \AA$ radiance measured by TIP during this orbit. We observe asymmetric peaks in the Appleton anomalies with the southern ionization crest being about $33 \%$ brighter than the northern one.

The GPS occultation data from this orbit are used to derive profiles of electron density along the FORMOSAT-3/ COSMIC FM6 orbit. Occultation measurements are inverted using a tomographic algorithm based on discrete inverse theory (Dymond and Thomas 2001). Peak electron densities $(\mathrm{nmF} 2)$ from these derived profiles are computed and shown in Fig. 6. The nmF2 latitudinal profile derived from the tomographic inversion of GOX data appears similar to that of the TIP measured OI $1356 \AA$ intensities with asymmetric peaks in the ionization crests. Peak electron densities of about and $6.5 \times 10^{5}$ and $7.6 \times 10^{5} \mathrm{~cm}^{-3}$ are inferred in the northern and southern anomalies, respectively.

The horizontal gradients in the $1356 \AA$ intensity measured by TIP are computed as $\partial(4 \pi I) / 4 \pi I \partial \mathrm{x}$ and are shown in Fig. 7. The gradients in $\mathrm{nmF} 2$ derived from the GPS occultation data are calculated as $2 \partial n / n \partial \mathrm{x}$ and overlaid on the $1356 \AA$ gradient as the gray, dashed line in Fig. 7. Here we

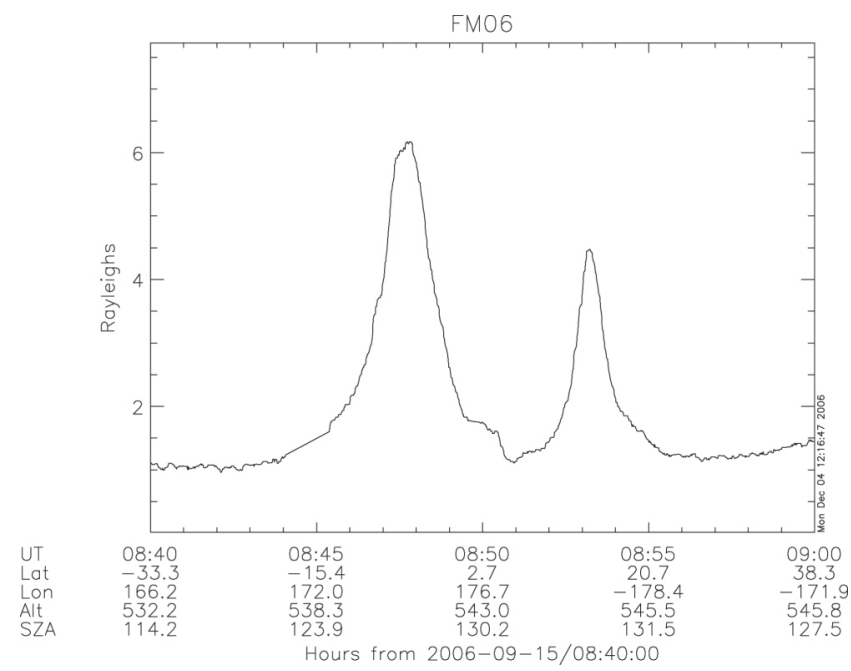

Fig. 5. OI $1356 \AA$ radiance measured by TIP on FORMOSAT-3/COSMIC FM6 between 0840 - 0900 UT 15 September 2006. 


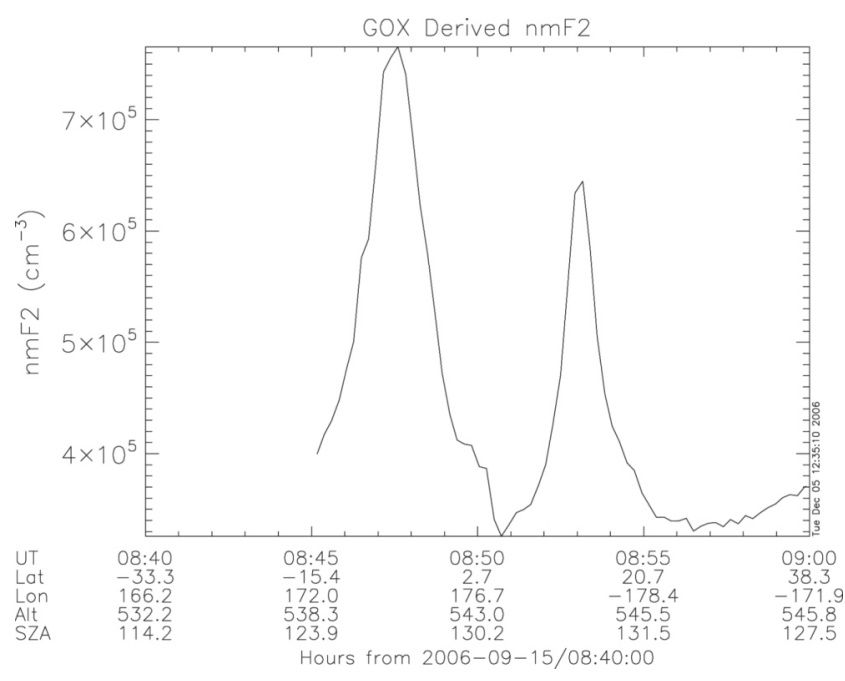

Fig. 6. NmF2 derived from tomographic inversion of FORMOSAT-3/ COSMIC GPS radio occultation data (GOX) for this same orbit on 15 September 2006.

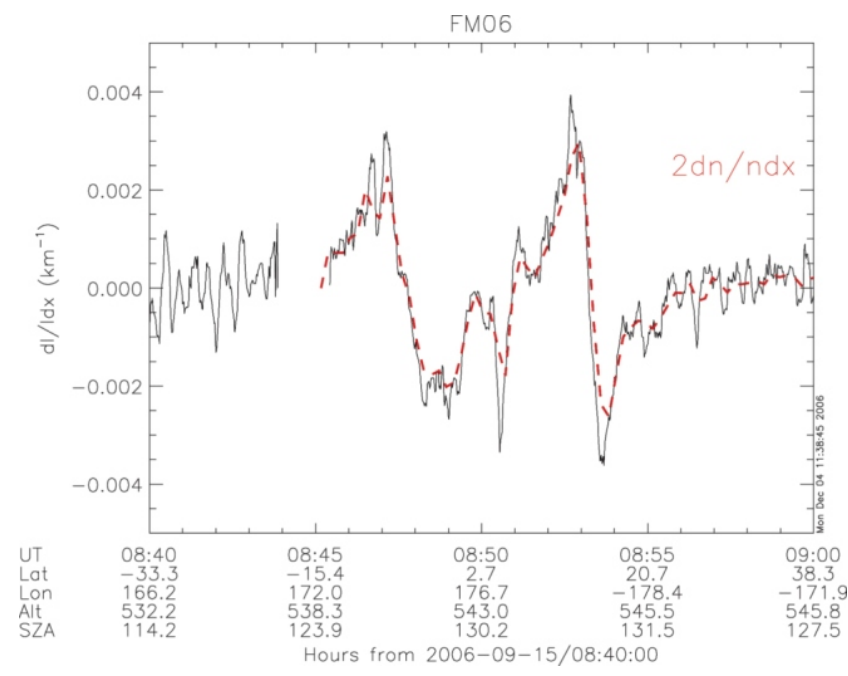

Fig. 7. Horizontal gradients in the TIP measured OI $1356 \AA$ radiance (solid) and the GOX derived nmF2 (dashed) for the FORMOSAT-3/ COSMIC FM6 orbit on 15 September 2006.

ignore the horizontal gradients in the $\mathrm{O}^{+}$scale height as we expect these variations to be small based on the results from our IRI-2001 model runs (section 3). Despite not taking the $\mathrm{O}^{+}$scale height term into account, the gradients in the $1356 \AA$ radiance observed by TIP track those of the peak electron densities inferred from the GPS occultation data very well. The largest deviations occur on either side of the peaks in the Appleton anomalies. Both the IRI-2001 model results and these data show that the second term on the right side of Eq. (4) is sufficiently small such that

$$
\frac{\partial(4 \pi I)}{4 \pi I \partial x} \approx \frac{2 \partial n_{e}}{n_{e} \partial x}
$$

This comparison validates our technique for tracking the horizontal variations in ionospheric electron density using measurements of the OI $1356 \AA$ radiance.

\section{VARIABILITY IN THE NIGHTSIDE IONOSPHERE}

We have shown that the FORMOSAT-3/COSMIC TIP measurements provide detailed measurements of the spatial variation of electron densities in the nightside ionosphere. In Fig. 8, we demonstrate that this spatial variability is captured over a broad range of longitudes by the FORMOSAT-3/ COSMIC constellation. Here, observations of the $1356 \AA$ radiance from the FORMOSAT-3/COSMIC FM1, FM3, FM5, and FM6 spacecraft are shown in this map projection of their ascending node passes throughout 17 September 2006 at local times between 18:00 and 21:00. Large longitudinal variations in the latitudinal separation, relative widths, and relative intensities of the northern and southern ionization crests of the Appleton anomalies are observed. Maxima in both intensity and latitudinal separation of the equatorial ionization anomalies occur in the Pacific, East Asia, and Africa sectors. The large-scale variations of the Appleton anomalies over distances greater than $1000 \mathrm{~km}$ are consistent with the longitudinal structuring observed by Immel et al. (2006) using IMAGE-FUV images of the nightside ionosphere. Immel et al. (2006) attribute these large-scale, global longitudinal variations in the equatorial anomalies to tidal forcing from the lower atmosphere.

The TIP measurements shown in Fig. 8 reveal smaller scale variations in the structure of the equatorial anomalies. In general, we observe non-conjugacy in the northern and southern ionization crests across the magnetic equator. This is especially apparent in the East Asia and Africa sectors where the southern ionization crests are observed to be about a factor of two or three more intense than the corresponding northern crests. The relative intensities of the northern and southern ionization crests vary more across the Pacific sector but in general the northern peaks appear to be more intense by as much as a factor of three.

A detailed examination of the TIP measurements on 17 September 2006 reveals the large degree of variability in the structure of the equatorial, nightside ionosphere. In Fig. 9, we show the $1356 \AA$ measurements from FORMOSAT-3/ COSMIC FM1 and FM5 which are in the East Asia and Kwajalein sectors respectively, separated by about $20^{\circ}$ in longitude. The TIP on FM5 observes highly asymmetric ionization crests of the Appleton anomaly in the Kwajalein sector where the northern peak is about a factor of three more intense than the southern peak (Fig. 9b). Large horizontal gradients in electron density are observed at the edges of the northern ionization crest (Fig. 9d). In contrast to the equatorial anomaly structure seen by FM5, FM1 observes a 
deep depletion in the electron densities as inferred by the factor of four drop-out in $1356 \AA$ radiance (Fig. 9a). The north-south scale size of this depletion is about $450 \mathrm{~km}$ and we interpret this to be an equatorial plasma bubble. The horizontal electron density gradients observed at the walls of this depletion are about a factor of two larger than those associated with the equatorial anomaly ionization crest observed by FM5 (Fig. 9c).

\section{VLF WAVE PROPAGATION IN THE PRESENCE OF HORIZONTAL ELECTRON DENSITY GRADIENTS}

Given the very different latitudinal profiles of the horizontal structure of the equatorial ionosphere at the two longitudes shown above, we expect a strong local time dependence for the characteristics of phenomena that are affected by electron density gradients such as radio wave propagation. For example, the propagation of both naturally occurring and artificially generated VLF waves has been shown to be influenced by horizontal gradients of electron density (James 1972; Fiszleiber et al. 1975; Cairó and Cerisier 1976). Fiszleiber et al. (1975) demonstrated a linear relationship between the components of a VLF wave's unit wave normal and the mean logarithmic horizontal electron density gradients. Cairó and Cerisier (1976) used measurements from the FR-1 satellite to show that the east-west and north-south components of VLF wave normal vectors were directly related to large-scale structures in the electron density profiles along each of those directions. Ray tracing studies have also found that horizontal electron density gradients associated with the equatorial anomalies lead to the focusing of ELF/ VLF whistler wave energy near the geomagnetic equator (Sonwalkar et al. 1995; Bortnik et al. 2002).

The strong longitudinal variations in horizontal electron density gradients observed by FORMOSAT-3/COSMIC TIP will modulate the ray paths of VLF whistler waves as a func- tion of local time. To investigate this effect, we examine the relationship between horizontal electron density gradients and the refractive index of VLF waves. A simple expression for the refractive index, $\mu$, for whistler-mode waves in a cold, collisionless plasma is given by Fiszleiber et al. (1975) as:

$\mu^{2} \cong 1+\frac{f_{p}^{2}}{f f_{c}|\cos \theta|-f^{2}}$

where $f$ is the wave frequency, $f_{p}$ is the plasma frequency, $f_{c}$ is the electron cyclotron frequency, and $\theta$ is the angle between the wave normal vector and the magnetic field direction. This equation is valid for:

$\frac{f}{f_{c}}<<|\cos \theta|$

For VLF waves $f \leq 30 \mathrm{kHz}$ and in the F-region $f_{c}$ is on the order of a few MHz. This limits the wave normal vector to angles less than or near $80^{\circ}$ with respect to the magnetic field. In the F-region plasma frequencies are on the order of tens of MHz such that $f_{p}^{2}>>f f_{c}>>f^{2}$, making the second term on the right hand side of Equation 6 much larger than unity. In this regime, we approximate Eq. (6) as:

$\mu^{2} \cong \frac{f_{p}^{2}}{f f_{c}|\cos \theta|}$

Substituting the definitions for the plasma and electron cyclotron frequencies (in SI units), we rewrite Eq. (8) in terms of the electron density as:

$\mu^{2} \cong \frac{n_{e} q_{e}}{f \varepsilon_{o} B|\cos \theta|}$

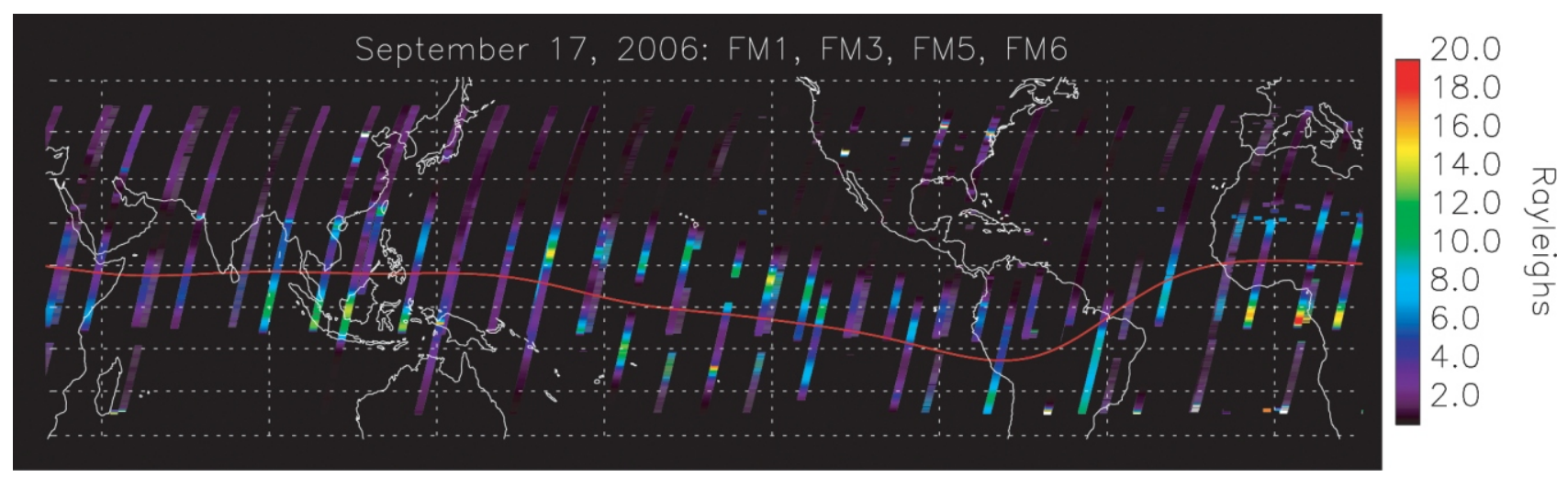

Fig. 8. Map view of TIP $1356 \AA$ Abservations from the FORMOSAT-3/COSMIC FM1, FM3, and FM5 spacecraft on 17 September 2006 showing the longitudinal variation in the variability in structure of the nightside ionosphere. The local time of these observations is between 18:00 - 21:00. The magnetic dip equator is shown in red. 

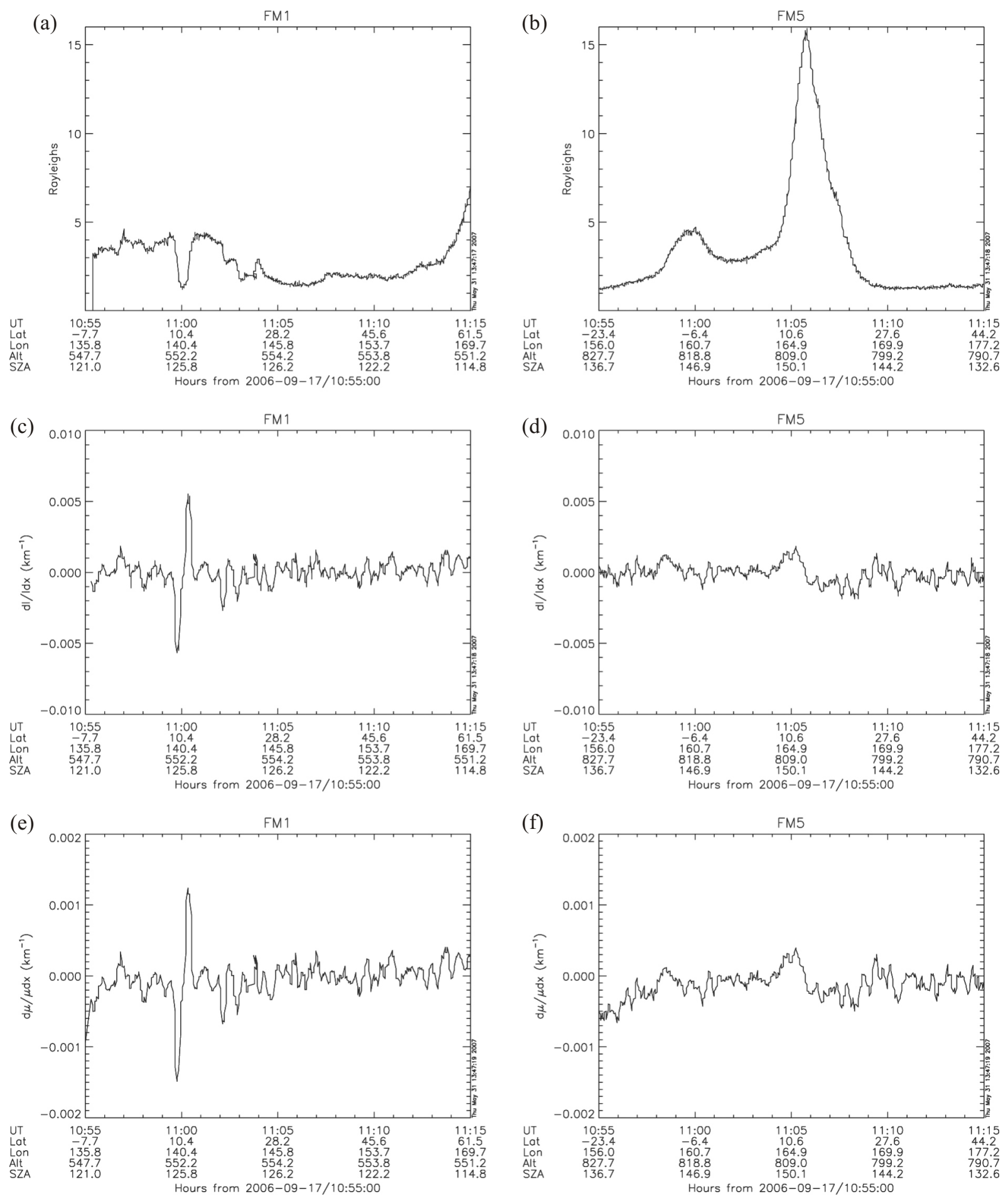

Fig. 9. (a) and (b) North-south profiles of OI $1356 \AA$ radiance measured by FORMOSAT-3/COSMIC FM1 and FM5 on 17 September 2006. (c) and (d) Inferred from these measurements are the electron density gradients, and (e) and (f) the gradients in the whistler-mode refractive index in the plane of the FORMOSAT-3/COSMIC FM1 and FM5 orbits.

where $q_{\mathrm{e}}$ is the electron charge, $\varepsilon_{\mathrm{o}}$ is the permittivity of free space, and $B$ is the magnetic field strength. The spatial derivative of Eq. (9) along the track of a FORMOSAT-3/COSMIC satellite gives the horizontal variation of the whistler-mode refractive index for a fixed frequency wave as:

$$
\frac{1}{\mu} \frac{\partial \mu}{\partial x}=\frac{1}{2}\left(\frac{1}{n_{e}} \frac{\partial n_{e}}{\partial x}-\frac{1}{B} \frac{\partial B}{\partial x}-\frac{1}{|\cos \theta|} \frac{\partial|\cos \theta|}{\partial x}\right)
$$


We use Eq. (5) to express Eq. (10) in terms of the horizontal $1356 \AA$ emission intensity gradient measured by TIP:

$$
\frac{1}{\mu} \frac{\partial \mu}{\partial x}=\frac{1}{2}\left[\frac{1}{2(4 \pi I)} \frac{\partial(4 \pi I)}{\partial x}-\frac{1}{B} \frac{\partial B}{\partial x}-\frac{1}{|\cos \theta|} \frac{\partial|\cos \theta|}{\partial x}\right]
$$

The horizontal variation of the whistler-mode refractive index is proportional to the horizontal gradients in $1356 \AA$ intensity or electron density, the horizontal variation in the magnetic field strength, and the horizontal variation in the relative angle of the wave normal vector to the magnetic field.

We examine the variation of the expected whistler-mode refractive index for the two FORMOSAT-3/COSMIC orbits shown in Fig. 9 using the horizontal electron density gradients measured by TIP. The magnetic field strength at an altitude of $300 \mathrm{~km}$ along the FM1 and FM5 orbits is computed using the IGRF 2000 model (Olsen et al. 2000). The third term on the right hand side of Eq. (10) is computed for obliquely propagating waves with an initial normal direction of $60^{\circ}$ from vertical. The expected horizontal gradients of the whistler-mode refractive indices along the orbits of the FORMOSAT-3/COSMIC FM1 and FM5 spacecraft are shown in Figs. 9e and f. Since the second and third terms of Eq. (10) vary less over the portions of the spacecraft orbits shown in Fig. 9 than the first term, the spatial variability in the refractive index gradients mostly reflects those of the inferred electron density gradients. We can immediately see that the variations of the whistler-mode refractive index shown in Fig. 9e will affect propagating ray paths very differently than those illustrated in Fig. 9f. The gradient in the whistler-mode refractive index across the boundaries of the plasma depletion observed by FM1 (Fig. 9e) is more than a factor of two greater than that observed by FM5 in the Appleton anomalies (Fig. 9f).

We have demonstrated that the horizontal variation of the whistler-mode refractive index can be measured by the FORMOSAT-3/COSMIC TIP observations. In continuing studies we will investigate the effects of horizontal electron density gradients on VLF wave propagation in more detail by performing ray tracing calculations using the refractive index gradients measured by TIP and numerical integration of the Haselgrove equations that describe the propagation of waves in a slowly varying medium (Haselgrove 1955). As an example, Fiszleiber et al. (1975) give the equation governing the variation of the north-south component of the ray path wave normal in spherical, polar coordinates $(r, \lambda, \phi)$ in the form:

$\frac{d\left(r \mu k_{\lambda}\right)}{d t}=\frac{\partial \mu}{\mu \partial \lambda}+r \mu k_{\phi} \cos \lambda \frac{d \phi}{d t}$ where $k_{\lambda}$ and $k_{\phi}$ are the latitudinal and longitudinal components of the wave normal vector, and $t$ is phase time. If we ignore longitudinal variations such that $k_{\phi}=0$ and expand the left-hand side, Eq. (12) becomes:

$\frac{d k_{\lambda}}{d t}=\frac{1}{\mu r} \frac{\partial \mu}{\mu \partial \lambda}-\frac{k_{\lambda}}{\mu} \frac{d \mu}{d t}-\frac{k_{\lambda}}{r} \frac{d r}{d t}$

The phase time is related to the path length as $d t=\mu \cos$ $\alpha d s$ where $\alpha$ is the angle between the wave normal and the ray path direction. In performing the ray tracing, we will use the TIP measurements to compute the first term in Eq. (12) or (13) via Eq. (11) given the appropriate coordinate transformation between the satellite orbit track and these spherical, polar coordinates.

\section{DISCUSSION AND CONCLUSIONS}

We have demonstrated a method of accurately determining the horizontal gradients in electron density using observations of OI $1356 \AA$ emission obtained from the FORMOSAT-3/COSMIC TIP instruments. The spatial variation in the nightside ionosphere captured by the TIP measurements in latitude and over many longitude sectors will be invaluable to models of ionospheric specification such as the Global Assimilation of Ionospheric Measurements (GAIM) model (Schunk et al. 2004) to better represent smaller scale structures that occur over short time scales. These TIP measurements and this technique for inferring the gradients in electron density will also improve studies of ionospheric irregularities and bubbles and the association of these phenomena with scintillations and wave propagation through these regions. In this study we began to describe how VLF wave propagation is affected by ionospheric structure by demonstrating how the horizontal gradients in the whistlermode refractive index can be computed using TIP measurements. In a proceeding study, we will offer a more detailed analysis of how ray paths are governed by the measured gradients in electron density observed by the FORMOSAT-3/COSMIC TIP instruments.

Acknowledgements The IRI-2001 model was provided by the NASA Goddard Space Flight Center Space Physics Data Facility (SPDF) through their Modelweb interface available at http://modelweb.gsfc.nasa.gov/models/iri.html.

\section{REFERENCES}

Aarons, J., R. S. Allen, and H. E. Whitney, 1972: Observations of scintillations of two satellite beacons near the boundary of the irregularity region. Planet. Spa. Sci., 20, 965, doi: 10.1016/0032-0633(72)90209-7. [Link]

Basu, S., 1974: VHF ionospheric scintillations at $\mathrm{L}=2.8$ and 
formation of stable auroral red arcs by magnetospheric heat conduction. J. Geophys. Res., 79, 3155, doi: 10.1029/ JA079i022p03155. [Link]

Basu, S. and S. Basu, 1985: Equatorial scintillations: Advances since ISEA-6. J. Atmos. Terr. Phys., 47, 753-768, doi: 10.1016/0021-9169(85)90052-2. [Link]

Basu, S., S. Basu, C. E. Valladares, H. C. Yeh, S. Y. Su, E. MacKenzie, P. J. Sultan, J. Aarons, F. J. Rich, P. Doherty, K. M. Groves, and T. W. Bullett, 2001: Ionospheric effects of major magnetic storms during the international space weather period of September and October 1999: GPS observations, VHF/UHF scintillations, and in situ density structures at middle and equatorial latitudes. J. Geophys. Res., 106, 30389-30413, doi: 10.1029/2001JA001116. [Link]

Bhattacharyya, A., T. L. Beach, S. Basu, and P. M. Kintner, 2000: Nighttime equatorial ionosphere: GPS scintillations and differential carrier phase fluctuations. Radio Sci., 35, 209-224, doi: 10.1029/1999RS002213. [Link]

Bilitza, D., 2001: International reference ionosphere 2000. $R a$ dio Sci., 36, 261-275, doi: 10.1029/2000RS002432. [Link]

Bortnik, J., U. S. Inan, and T. F. Bell, 2002: L dependence of energetic electron precipitation driven by magnetospherically reflecting whistler waves. J. Geophys. Res., 107, 1150, doi: 10.1029/2001JA000303. [Link]

Cairó, L. and J. C. Cerisier, 1976: Experimental study of ionospheric electron density gradients and their effect on VLF propagation. J. Atmos. Terr. Phys., 38, 27-36, doi: 10.1016/ 0021-9169(76)90190-2. [Link]

Coker, C., K. F. Dymond, S. A. Budzien, D. H. Chua, J. Y. Liu, D. N. Anderson, S. Basu, and T. R. Pedersen, 2009: Observations of the ionosphere using the Tiny Ionospheric Photometer. Terr. Atmos. Ocean. Sci., 20, 227-235, doi: 10.3319/ TAO.2008.01.18.02(F3C). [Link]

Dymond, K. F. and R. J. Thomas, 2001: A technique for using measured ionospheric density gradients and GPS occultations for inferring the nighttime ionospheric electron density. Radio Sci., 36, 1141-1148, doi: 10.1029/2000RS 002430. [Link]

Dymond, K. F., S. E. Thonnard, R. P. McCoy, and R. J. Thomas, 1997: An optical remote sensing technique for determining nighttime F region electron density. Radio Sci., 32, 19851996, doi: 10.1029/97RS01887. [Link]

Dymond, K. F., J. B. Nee, and R. J. Thomas, 2000: The Tiny Ionospheric Photometer: An instrument for measuring ionospheric gradients for the COSMIC constellation. Terr. Atmos. Ocean Sci., 11, 273-290.

Dymond, K. F., S. A. Budzien, D. H. Chua, C. Coker, and J. Y. Liu, 2009: Tomographic reconstruction of the low-latitude nighttime electron density using FORMOSAT-3/COMSIC radio occultation and UV photometer data. Terr. Atmos.
Ocean. Sci., 20, 215-226, doi: 10.3319/TAO.2008.01.15. 01(F3C). [Link]

Fiszleiber, F., H. de Feraudy, and J. C. Cerisier, 1975: Influence of horizontal density gradients on the propagation of VLF waves through the ionosphere. J. Atmos. Terr. Phys., 37, 1525-1534, doi: 10.1016/0021-9169(75)90031-8. [Link]

Haselgrove, J., 1955: Ray theory and a new method for ray tracing, in Physics of the Ionosphere, Physical Society, London, 355-364.

Immel, T. J., E. Sagawa, S. L. England, S. B. Henderson, M. E. Hagan, S. B. Mende, H. U. Frey, C. M. Swenson, and L. J. Paxton, 2006: Control of equatorial ionospheric morphology by atmospheric tides. Geophys. Res. Lett., 33, L15108, doi: 10.1029/2006GL026161. [Link]

James, H. G., 1972: Refraction of whistler-mode waves by large-scale gradients in the middle-latitude ionosphere. Ann. Geophys., 2, 301-339.

Kalmanson, P. C., S. A. Budzien, C. Coker, and K. F. Dymond, 2004: The Tiny Ionospheric Photometer instrument design and operation. Proc. SPIE, 5660, 259-270, doi: 10.1117/ 12.578341. [Link]

Ledvina, B. M., P. M. Kintner, and J. J. Makela, 2004: Temporal properties of intense GPS L1 amplitude scintillations at midlatitudes. Radio Sci., 39, doi: 10.1029/2002RS002832. [Link]

Meléndez-Alvira, D. J., R. R. Meier, J. M. Picone, P. D. Feldman, and B. M. McLaughlin, 1999: Analysis of the oxygen nightglow measured by the Hopkins Ultraviolet Telescope: Implications for ionospheric partial radiative recombination rate coefficients. J. Geophys. Res., 104, 14901-14913, doi: 10.1029/1999JA900136. [Link]

Olsen, N., T. J. Sabaka, and L. Tøffner-Clausen, 2000: Determination of the IGRF 2000 model. Earth Planets Space, 52, $1175-1182$.

Rocken, C., Y. H. Kuo, W. Schreiner, D. Hunt, and S. Sokolovskiy, 2000: COSMIC system description. Terr. Atmos. Ocean Sci., 11, 21-52.

Schunk, R. W., L. Scherliess, J. J. Sojka, D. Thompson, D. N. Anderson, M. Codrescu, C. Minter, T. J. Fuller-Rowell, R. A. Heelis, M Hairston, and B. M. Howe, 2004: Global Assimilation of Ionospheric Measurements (GAIM). Radio Sci., 39, RS1S02, doi: 10.1029/2002RS002794. [Link]

Sonwalkar, V. S., U. S. Inan, T. L. Aggson, W. M. Farrell, and R. Pfaff, 1995: Focusing of nonducted whistlers by the equatorial anomaly. J. Geophys. Res., 100, 7783-7790, doi: 10.1029/94JA02756. [Link]

Valladares, C. E., J. Villalobos, R. Sheehan, and M. P. Hagan, 2004: Latitudinal extension of low-latitude scintillations measured with a network of GPS receivers. Ann. Geophys., 22, 3155-3175. 